\title{
Whole genome shotgun sequence of Bacillus amyloliquefaciens TF28, a biocontrol entophytic bacterium
}

\author{
Shumei Zhang ${ }^{1,2}$, Wei Jiang ${ }^{1,2}$, Jing $\mathrm{Li}^{1,2}$, Liqiang Meng ${ }^{1,2}, \mathrm{Xu} \mathrm{CaO}^{1,2}$, Jihua $\mathrm{Hu}^{2}$, Yushuai Liu ${ }^{1,2}$, Jingyu Chen² \\ and Changqing Sha $a^{2,3^{*}}$
}

\begin{abstract}
Bacillus amyloliquefaciens TF28 is a biocontrol endophytic bacterium that is capable of inhibition of a broad range of plant pathogenic fungi. The strain has the potential to be developed into a biocontrol agent for use in agriculture. Here we report the whole-genome shotgun sequence of the strain. The genome size of B. amyloliquefaciens TF2 8 is 3,987,635 bp which consists of 3754 protein-coding genes, 65 tandem repeat sequences, 47 minisatellite DNA, 2 microsatellite DNA, 63 tRNA, 7rRNA, 6 sRNA, 3 prophage and CRISPR domains.
\end{abstract}

Keywords: Genome sequence, Bacillus amyloliquefaciens, Endophytic bacterium, Biocontrol, Broad spectrum Abbreviations: gyrA, DNA gyrase subunit A; rpoB, RNA polymerase subunit B; TCA, Tricarboxylic acid cycle

\section{Introduction}

Bacillus amyloliquefaciens is ubiquitous in nature. Some strains are used as biocontrol agents because of their ability to produce antagonistic metabolites, plant growth promoters and plant health enhancers [1-4]. B. amyloliquefaciens is usually divided into two subspecies by genome comparison and classical bacterial taxonomy. Plant growth-promoting rhizobacterial strains are classified as $B$. amyloliquefaciens subsp. plantarum, while other strains are regarded as B. amyloliquefaciens subsp. amyloliquefaciens [5]. B. amyloliquefaciens TF28 is an endophytic bacterium that was isolated from soybean root. Previous studies have shown that $B$. amyloliquefaciens TF28 could inhibit soil borne and air borne plant pathogenic fungi by competition, synthesizing antifungal metabolites and inducing systemic plant resistance [6, 7]. Based on $16 \mathrm{~S}$ rRNA, DNA gyrase subunit A ( $g y r A)$ and RNA polymerase subunit $\mathrm{B}(r p o B)$ gene sequence analysis, $B$. amyloliquefaciens TF28 was classified as $B$. amyloliquefaciens subsp. plantarum. Here we present a whole-genome shotgun sequence of $B$. amyloliquefaciens TF28 and its

\footnotetext{
* Correspondence: shachangqing@163.com

${ }^{2}$ Institute of Advanced Technology, Heilongjiang Academy of Sciences, Harbin 150020, China

${ }^{3}$ Heilongjiang Academy of Sciences, Harbin 150001, China

Full list of author information is available at the end of the article
}

annotation for facilitating its application in the biocontrol of plant diseases.

\section{Organism information \\ Classification and features}

B. amyloliquefaciens TF28 was isolated from soybean root in China. It exhibited an unusual ability to inhibit a wide range of plant pathogenic fungi. The cell morphology of strain TF28 was determined using scanning electron microscopy (Fig. 1). Cells of B. amyloliquefaciens TF28 are Gram-positive, rod shape, aerobic and endosporeforming. Strain TF28 utilizes glucose and lactose to produce acid and hydrolyzed gelatin and starch. Starin TF28 is positive for Vogues-Proskaur and Methyl red reaction, nitrate reduction and citrate utilization. Current taxonomic classification and general features of $B$. amyloliquefaciens TF28 are provided in Table 1.

The 16S rRNA gene sequence of strain TF28 and other available $16 \mathrm{~S}$ rRNA gene sequences of closely related species collected from NCBI database were used to construct a phylogenetic tree (Fig. 2, Additional file 1: Table S1). The evolutionary history was inferred using the Neighbour-joining method with MEGA software version 5.10. BLAST analysis showed strain B. amyloliquefaciens TF28 shared 99.3-99.7 \% 16S rRNA gene identities with the other 14 type strains of Bacillus species. Taxonomic 


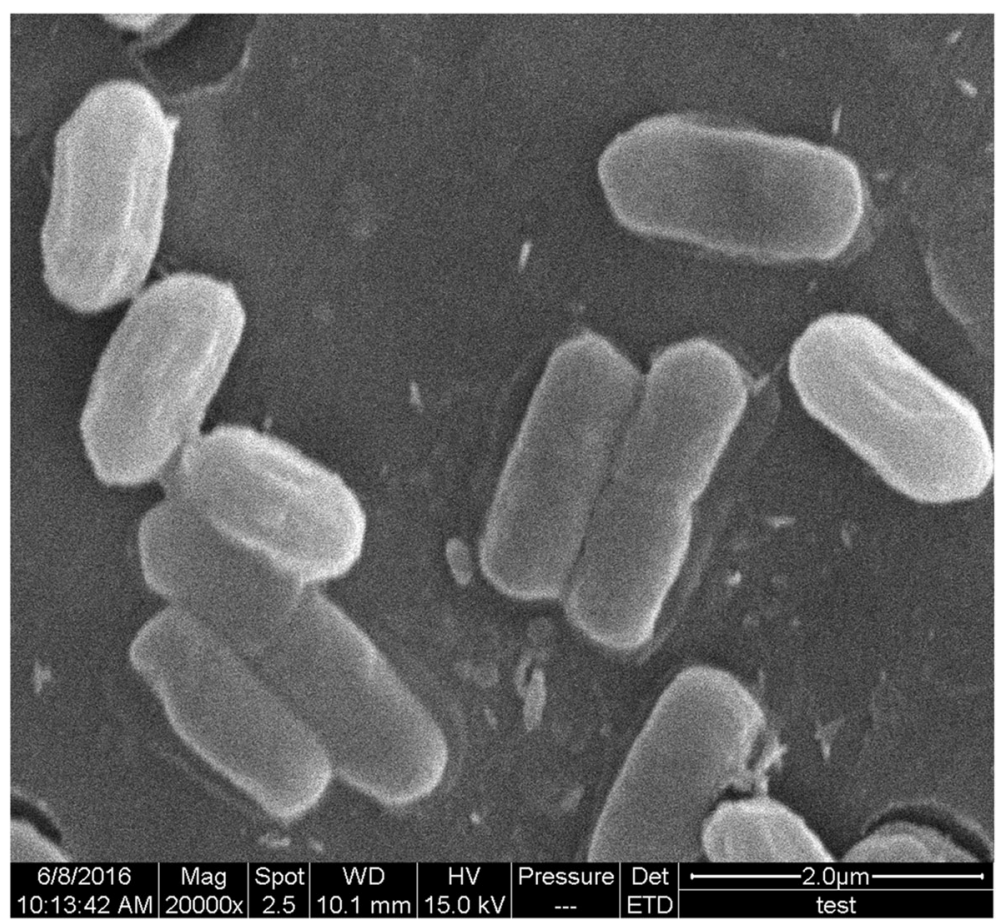

Fig. 1 A scanning electron micrograph of B. amyloliquefaciens TF 28 cells

analysis showed that 14 type strains were divided into two groups. Strain TF28 together with B. amyloliquefaciens subsp. plantarum $\mathrm{FZB} 42^{\mathrm{T}}$, B. methylotrophicus CBMB205 ${ }^{\mathrm{T}}$, B. amyloliquefaciens subsp. amyloliquefaciens $\mathrm{DSM}^{\mathrm{T}}$ and $B$. siamensis $\mathrm{PD}-\mathrm{A} 10^{\mathrm{T}}$ were clustered into one group. Other strains (B. atrophaeus NBRC $15539^{\mathrm{T}}$, B. vallismortis DSM $11031^{\mathrm{T}}$, B. tequilensis $10 \mathrm{~b}^{\mathrm{T}}$, B. subtilis $168^{\mathrm{T}}$, B. subtilis subsp. subtilis DSM $10^{\mathrm{T}}$, B. subtilis subsp. inaquosorum BGSC $3 \mathrm{~A} 28^{\mathrm{T}}$, B. subtilis subsp. spizizenii NBRC $101239^{\mathrm{T}}$, B. mojavensis NBRC $15718^{\mathrm{T}}$, B. malacitensis CR- $95^{\mathrm{T}}$ and B. axarquiensis LMG $22476^{\mathrm{T}}$ ) were clustered into another group. Two type stains of $B$. amyloliquefaciens subspecies, B. amyloliquefaciens B. amyloliquefaciens subsp. plantarum $\mathrm{FZB} 42^{\mathrm{T}}$ and B. amyloliquefaciens subsp. amyloliquefaciens $\mathrm{DSM}^{\mathrm{T}}$ were attributed to the different clade. Strain TF28 was most closely related to B. amyloliquefaciens subsp. plantarum $\mathrm{FZB} 42^{\mathrm{T}}$ with $99.7 \% 16 \mathrm{~S}$ rRNA gene sequence identity. Strain TF28 was classified as B. amyloliquefaciens subsp. plantarum.

\section{Genome sequencing information \\ Genome project history}

Genome of B. amyloliquefaciens TF28 was sequenced by Huada Gene Technology Co., Ltd, Shenzhen, China. The Whole Genome Shotgun sequence has been deposited in GenBank database under the accession number JUDU00000000. The summary of the project information is shown in Table 2.
Growth conditions and genomic DNA preparation

B. amyloliquefaciens TF28 was grown in LB medium at $30{ }^{\circ} \mathrm{C}$ for $16 \mathrm{~h}$. One liter cultures at the exponential growth phase was taken and centrifuged at $4{ }^{\circ} \mathrm{C}$, $5000 \mathrm{rpm}$ for $10 \mathrm{~min}$. The pellet was collected and about $5 \mathrm{~g}$ cell pellet was used to extract genomic DNA by CTAB method [8]. The quality of DNA was assessed using a Qubit Fluorometer. Total DNA $(280.6 \mu \mathrm{g})$ was obtained to do genome sequencing.

\section{Genome sequencing and assembly}

Genomic DNA was sheared randomly. The required length DNA fragments were retained by electrophoresis and used for construction of a $500 \mathrm{bp}$ and $6000 \mathrm{bp}$ long paired-end library. Sequencing was performed by Illumina HiSeq 2000 sequencing platform. Sequencing of the 500 bp library generated 6,649,820 reads (representing $554 \mathrm{Mbp}$ of sequence information), while sequencing of the 6,000 bp paired-end library generated 3,633,388 reads (290 Mbp). Both libraries achieved a genome coverage of $190 \times$ for an estimated genome size of 4.4 Mbp. All generated reads were quality trimmed to obtain clear reads. The trimmed reads were assembled by SOAPdenovo software 2.04 using the available genome sequence of $B$. amyloliquefaciens subsp. plantarum $\mathrm{FZB} 42^{\mathrm{T}}$ (CP000560) as reference-guided assembling. The final assembly yielded 182 contigs and 3 scaffolds representing 3.9 Mbp of sequence information. 
Table 1 Classification and general features of B.amyloliquifaciens TF28 as per MIGS recommendation [26]

\begin{tabular}{|c|c|c|c|}
\hline MIGS ID & Property & Term & Evidence code $^{a}$ \\
\hline & Classification & Domain Bacteria & TAS [27] \\
\hline & & Phylum Firmicutes & TAS [28-30] \\
\hline & & Class Bacilli & TAS $[31,32]$ \\
\hline & & Order Bacillales & TAS $[29,33]$ \\
\hline & & Family Bacillaceae & $\operatorname{TAS}[29,34]$ \\
\hline & & Genus Bacillus & $\operatorname{TAS}[29,35]$ \\
\hline & & Species Bacillus amyloliquefaciens & TAS [36-38] \\
\hline & & Strain: TF28 & TAS [6] \\
\hline & Gram stain & Positive & TAS [6] \\
\hline & Cell shape & Rod & TAS [6] \\
\hline & Motility & Motile & TAS [6] \\
\hline & Sporulation & Endospore-forming & TAS [6] \\
\hline & Temperature range & $15-37^{\circ} \mathrm{C}$ & TAS [6] \\
\hline & Optimum temperature & $30^{\circ} \mathrm{C}$ & TAS [6] \\
\hline & pH range; Optimum & $5-9,7.5$ & TAS [6] \\
\hline & Carbon source & Glucose, lactose, starch & TAS [6] \\
\hline MIGS-6 & Habitat & Soil, Plant & TAS [6] \\
\hline MIGS-6.3 & Salinity & $0-3 \%$ WN & TAS [6] \\
\hline MIGS-22 & Oxygen requirement & Aerobic & TAS [6] \\
\hline MIGS-15 & Biotic relationship & Free-living & TAS [6] \\
\hline MIGS-14 & Pathogenicity & Non-pathogen & NAS \\
\hline MIGS-4 & Geographic location & China/Heilongjiang & TAS [6] \\
\hline MIGS-5 & Sample collection & 2006-06-10 & TAS [6] \\
\hline MIGS-4.1 & Latitude & Not reported & \\
\hline MIGS-4.2 & Longitude & Not reported & \\
\hline MIGS-4.4 & Altitude & Not reported & \\
\hline
\end{tabular}

aEvidence codes - NAS: Non-traceable Author Statement (i.e., not directly observed for the living, isolated sample, but based on a generally accepted property for the species, or anecdotal evidence). These evidence codes are from the Gene Ontology project [39]

\section{Genome annotation}

The genome sequence was annotated by a combination of several annotation tools. Genes were identified by Glimmer 3.02 [9]. DNA tandem repeat sequences, minisatellite DNA and microsatellite DNA were found with the Tandem Repeats Finder 4.04 [10]. Prediction of non-coding RNA was performed using rRNA database blasting or rRNAmmer 1.2 for rRNA [11], tRNAscan-SE 1.23 for tRNA and their secondary structure [12], and infernal software and Rfam database for sRNA [13]. Prophage was predicted using PHAST software 2013.03.20 [14]. CRISPR domains were found using CRISPR Finder 0.4 [15]. Functional annotation of protein coding genes was based on gene comparisons with GO database (version 1.419) [16], KEGG database (version 59) [17], Cluster of Orthologous Groups of proteins(COG)(version 20090331) [18], NR database(version 20121005), SwissProt (version 201206) [19] and Pfam databases (version 25) [20].

\section{Genome properties}

The genome statistics are provided in Table 3 and Fig. 3. The high quality draft genome of $B$. amyloliquefaciens TF28 was distributed in 182 contigs with a total size of $3,987,635$ bp and an average $\mathrm{G}+\mathrm{C}$ content of $46.38 \%$. Genome analysis showed that the genome of strain TF28 contained 3,754 protein coding genes, 65 tandem repeat sequences, 47 minisatellite DNA, 2 microsatellite DNA, 63 tRNA, 7 rRNA, 6 sRNA, 3 prophage and 3 CRISPR domains. The predicted protein coding genes represented $89.57 \%$ of the total genome sequence, with a total length of $3,571,596 \mathrm{bp}$. The majority of protein coding genes $(76.13 \%)$ were assigned to putative functions. The distribution of genes into COG functional categories is presented in Table 4.

\section{Insights from the genome sequence}

Protein coding genes were mainly classified into 3 parts based on their functions by GO analysis (Fig. 4). 1901, 


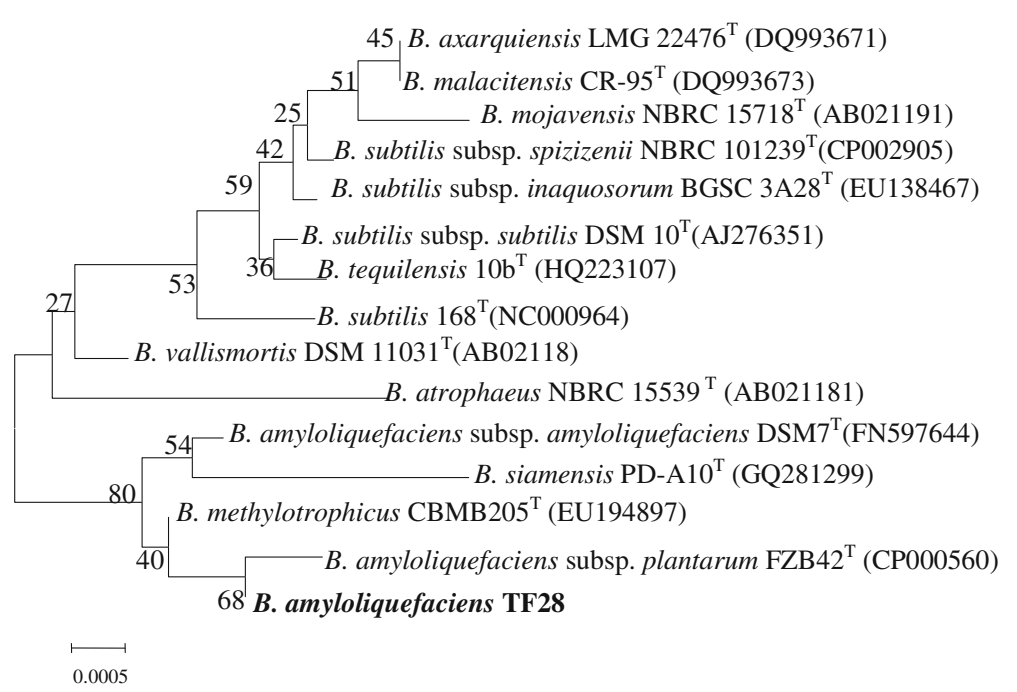

Fig. 2 Phylogenetic trees based on 165 rRNA gene sequences highlighting the position of B. amyloliquefaciens TF 28 (shown in bold). The GenBank accession numbers are shown in parentheses. Sequences were aligned using CLUSTALW, and phylogenetic inferences were constructed using the neighbor-joining method within the MEGA 5.10 software (Additional file 7: Table S7). Numbers at the nodes represent percentages of bootstrap values obtained by repeating the analysis 1000 times to generate a majority consensus tree. The scale bar indicates 0.0005 nucleotide change per nucleotide position, respectively

2993 and 4309 genes participated in cellular component, molecular function and biological process, respectively. The metabolic pathway analysis using KEGG annotation showed that the majority of protein coding genes participated in metabolism, genetic information processing, environmental information processing and cellular processes (Fig. 5). 154 metabolic pathways were found using KEGG orthology, including glycolysis, TCA cycle and pentose phosphate pathways, fructose, mannose and galactose metabolisms pathways, fatty acid biosynthesis and metabolism pathways, ubiquinone and other terpenoid-

Table 2 Project information

\begin{tabular}{lll}
\hline MIGS ID & Property & Term \\
\hline MIGS 31 & Finishing quality & High-quality draft \\
MIGS-28 & Libraries used & $\begin{array}{l}\text { Illumina Paired-End Library } \\
\text { (500 bp insert size) and Mate } \\
\text { Pair Library (6,000 bp insert size) }\end{array}$ \\
MIGS 29 & Sequencing platforms & Illumina Hiseq2000 \\
MIGS 31.2 & Fold coverage & 150x \\
MIGS 30 & Assemblers & SOAPdenovo software 2.04 \\
MIGS 32 & Gene calling method & Glimmer \\
& Locus Tag & TH57 \\
& GenBank ID & JUDU00000000 \\
& GenBank Date of Release & 2015/01/21 \\
& GOLD ID & - \\
& BIOPROJECT & PRJNA268537 \\
MIGS 13 & Source Material Identifier & TF28 \\
& Project relevance & Biocontrol, Agriculture \\
\hline
\end{tabular}

uquinoid synthesis pathways, bacterial chemotaxis, biosynthsis of siderophore group nonribosomal peptides, antibiotic biosynthesis (tetracycline, penicillin and cephalosporin, streptomycin, novobiocin and vancomycin) as well as noxious substance degradation pathways (caprolactam, atrazine, ethylbenzene, toluene, polycyclic aromatic hydrocarbon, chloroalkane and chloroalkene, bisphenol, naphthalene, aminobenzoate, limonene and pinene), and so on.

Genome similarity was detected based on Mummer blast by comparing the genome sequence of strain TF2 8 with the type strain $B$. amyloliquefaciens subsp. plantarum FZB42 ${ }^{\mathrm{T}}$ at amino acid level [21]. The results showed that genome similarity of $B$. amyloliquefaciens TF28 and $B$. amyloliquefaciens subsp. plantarum $\mathrm{FZB} 42^{\mathrm{T}}$ reached $98.69 \%$. Core-pan gene was also determined based on NCBI blast and Muscle analysis [22]. 201 strain-specific genes for B. amyloliquefaciens TF28 was observed, which may contribute to species-specific features of this bacterium. Among them, 83 genes are classified into 17 COG functional categories major belonging to carbohydrate transport and metabolism (6.97\%), general function prediction only (4.48\%), defense mechanisms (4.48\%), signal transduction mechanisms (3.48\%), amino acid transport and metabolism (3.98\%). The remaining 116 unique genes $(57.71 \%)$ are not classified into any COG categories (Table 5). Comparative genome analysis revealed that $B$. amyloliquefaciens TF28 possessed the giant gene clusters for non-ribosomal synthesis of the polyketides difficidin (TH57_02955-TH57_03045) and bacillaene (TH57_05575-TH57_05655), the antifungal 
Table 3 Genome statistics

\begin{tabular}{llc}
\hline Attribute & Value & \% of Total \\
\hline Genome size (bp) & $3,987,635$ & 100.00 \\
DNA coding (bp) & $3,571,596$ & 89.57 \\
DNA G + C (bp) & $1,849,465$ & 46.38 \\
DNA scaffolds & 3 & - \\
Total genes & 3863 & 100.00 \\
Protein coding genes & 3754 & 97.18 \\
RNA genes & 76 & 1.97 \\
Pseudo genes & 38 & 0.98 \\
Genes in internal clusters & 1554 & 40.23 \\
Genes with function prediction & 2941 & 76.13 \\
Genes assigned to COGs & 3218 & 83.30 \\
Genes with Pfam domains & 3292 & 85.21 \\
Genes with signal peptides & 204 & 5.28 \\
Genes with transmembrane helices & 1041 & 26.95 \\
CRISPR repeats & 3 & - \\
\hline
\end{tabular}

lipopetides surfactin (TH57_12375-TH57_12430), plipastatin (TH57_04780-TH57_04835), mycosubtilin (TH5704955-TH57-04980), bacilysin (TH57_15685-TH57_15710) and bacillibactin (TH57_05755-TH57_05800) (Additional file 2: Table S2). The size of these gene clusters accounted for $6.8 \%$ of genome, which was smaller than that of strain $\mathrm{FZB}_{2}{ }^{\mathrm{T}}(8.9 \%)$ [23]. Mycosubtilin and plipastatin synthesis gene clusters were only observed in strain TF28. These gene clusters produce the secondary metabolites like NRPSs, PKS, and peptide antibiotics usually displaying antifungal and antibacterial activities [23-25]. The finding of these gene clusters revealed that strain TF28 possessed a high potential to biocontrol. In addition, sporulation genes, spoOABFJ(TH57_02695,01435,16015and14190), spoVABCDEFKSMRT(TH57_03250,03255,03260,03265, 03270,03275,03280),SpoIIBPMERDQSASB(TH57_05470, 00570,06315,09520,13850),spoIIIABCDEFGH(TH57_01370, 02065,03200,07810,07815,13800,16095,16205and16305), coaX(TH57_01485),YtrIH(TH57_00725,009730),ylbJB(TH57_ 06685),ydcC(TH57_11735),ydhD(TH57_07580),cse15 (TH57_07135), yunB(TH57_18555) and motility

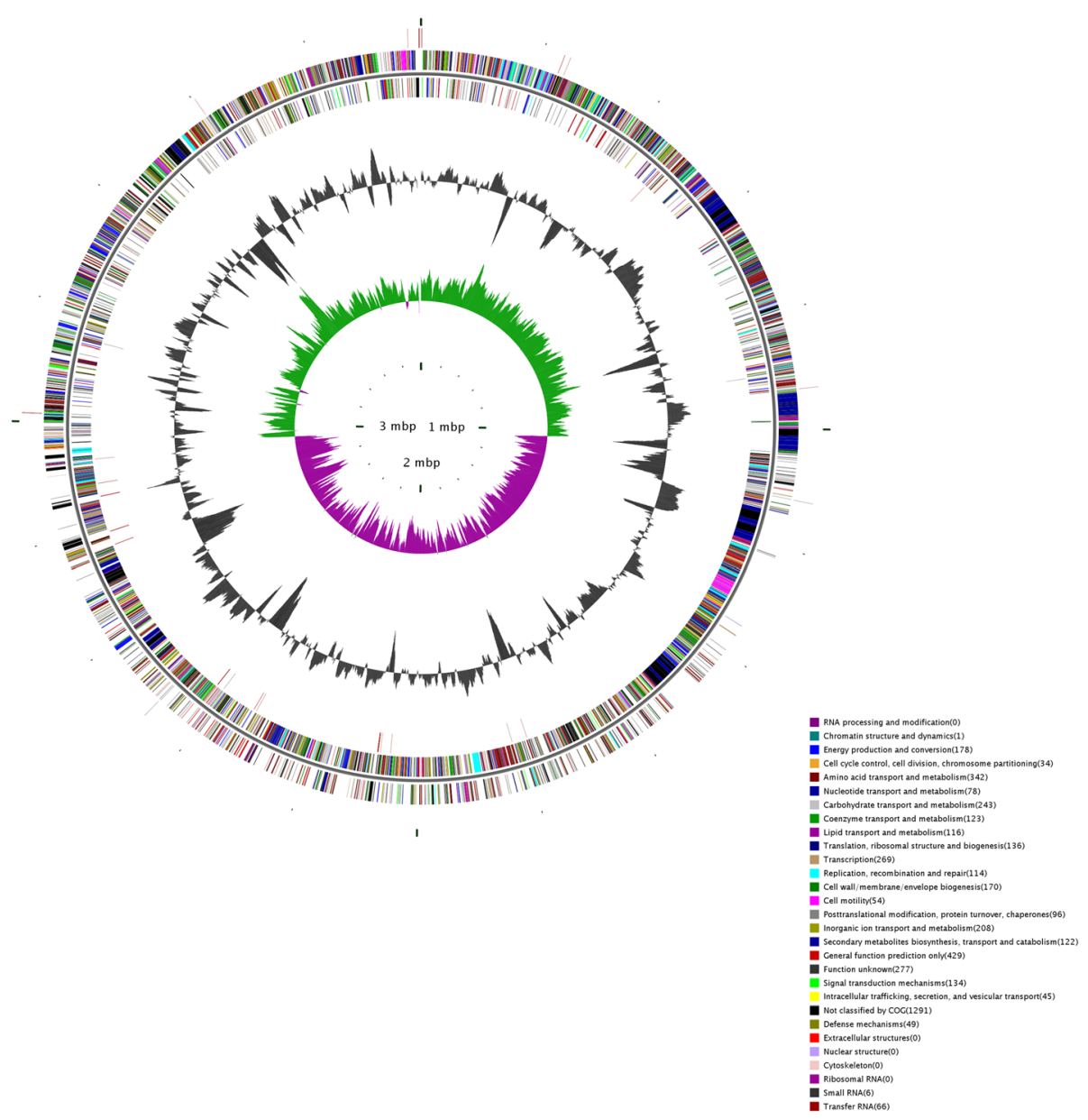

Fig. 3 Circle map of strain TF28 genome. From outer to inner circle, circle 1 shows protein-coding genes colored by COG categories; circle 2 shows G + C\% content plot; circle 3 shows GC skew 
Table 4 Number of genes associated with general COG functional categories

\begin{tabular}{|c|c|c|c|}
\hline Code & Value & \%age & Description \\
\hline J & 136 & 3.02 & Translation, ribosomal structure and biogenesis \\
\hline A & 0 & 0 & RNA processing and modification \\
\hline K & 269 & 5.97 & Transcription \\
\hline L & 114 & 2.53 & Replication, recombination and repair \\
\hline B & 1 & 0.02 & Chromatin structure and dynamics \\
\hline $\mathrm{D}$ & 34 & 0.75 & Cell cycle control, Cell division, chromosome partitioning \\
\hline V & 49 & 1.09 & Defense mechanisms \\
\hline $\mathrm{T}$ & 134 & 2.97 & Signal transduction mechanisms \\
\hline M & 170 & 3.77 & Cell wall/membrane biogenesis \\
\hline $\mathrm{N}$ & 54 & 1.19 & Cell motility \\
\hline U & 45 & 0.99 & Intracellular trafficking and secretion \\
\hline $\mathrm{O}$ & 96 & 2.13 & Posttranslational modification, protein turnover, chaperones \\
\hline C & 178 & 3.95 & Energy production and conversion \\
\hline G & 243 & 5.39 & Carbohydrate transport and metabolism \\
\hline $\mathrm{E}$ & 342 & 7.58 & Amino acid transport and metabolism \\
\hline $\mathrm{F}$ & 78 & 1.73 & Nucleotide transport and metabolism \\
\hline $\mathrm{H}$ & 123 & 2.73 & Coenzyme transport and metabolism \\
\hline । & 116 & 2.57 & Lipid transport and metabolism \\
\hline $\mathrm{P}$ & 208 & 4.61 & Inorganic ion transport and metabolism \\
\hline Q & 122 & 2.71 & Secondary metabolites biosynthesis, transport and catabolism \\
\hline $\mathrm{R}$ & 429 & 9.51 & General function prediction only \\
\hline S & 277 & 6.14 & Function unknown \\
\hline- & 1291 & 28.63 & Not in COGs \\
\hline
\end{tabular}

The total $\%$ age is based on the total number of protein coding genes in the annotated genome

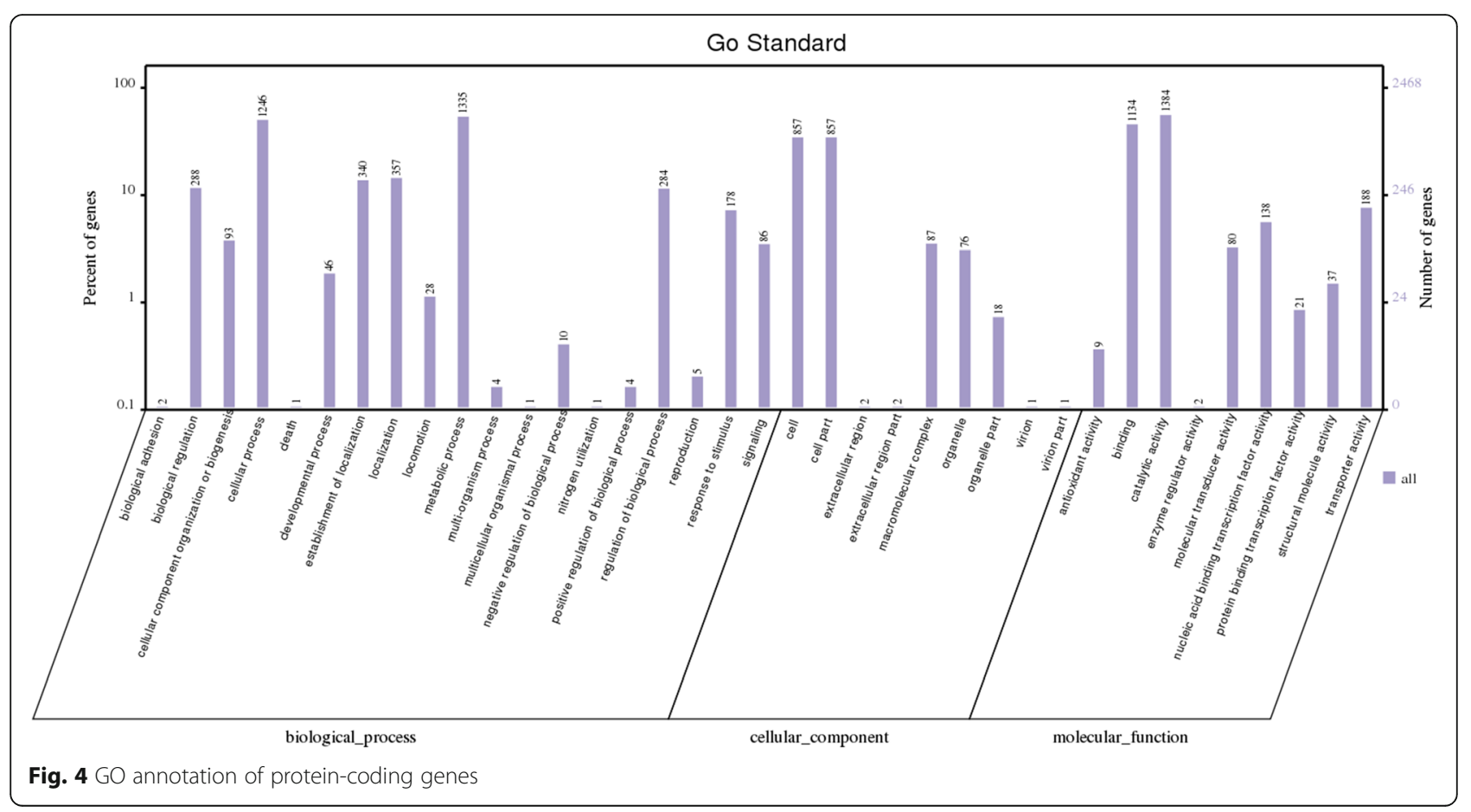




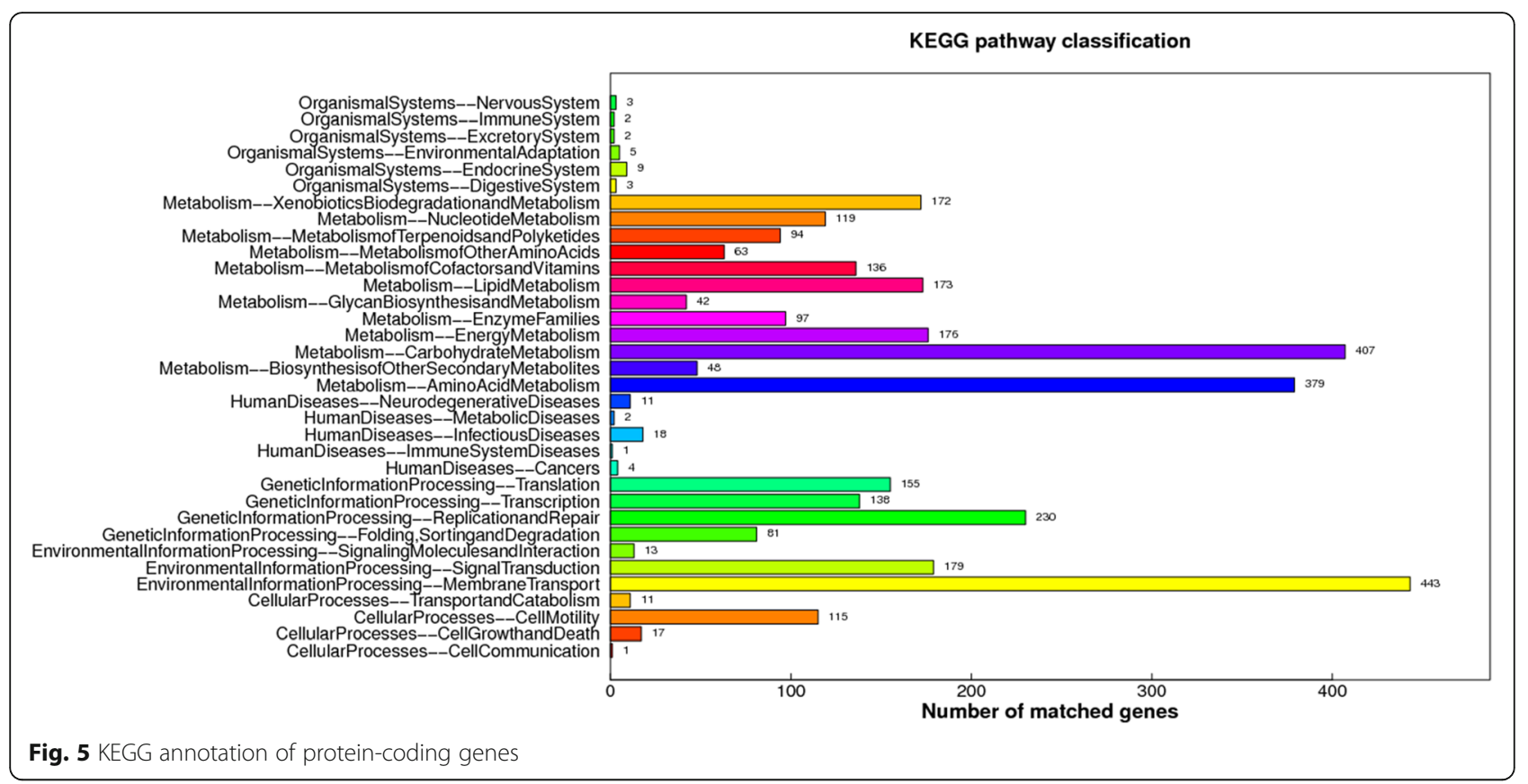

genes, motAB (TH57_074157, 07420) and swrABC (TH57_16980,05970 and 10760), were found in the genome.

Comparative genomic analysis of $B$. amyloliquefaciens TF28 and other 22 strains of B. amyloliquefaciens possessing complete genomic sequences indicated that the genome size of the strain TF28 was somewhat bigger than that of B. amyloliquefaciens subsp. plantarum $\mathrm{FZB} 2^{\mathrm{T}}$ and B. amyloliquefaciens subsp. amyloliquefaciens $\mathrm{DSM}^{\mathrm{T}}$. Three strains, B. amyloliquefaciens IT-45, B. amyloliquefaciens NAU-B3 and B. amyloliquefaciens TF28, possessed CRISPR domains by CRISPR Finder on line

Table 5 Number of strain-specific genes with general COG functional categories

\begin{tabular}{llll}
\hline Code & Value & \%age & Description \\
\hline A & 2 & 0.99 & Translation,ribosomal structure and biogenesis \\
K & 0 & 0 & RNA processing and modification \\
L & 5 & 2.49 & Transcription \\
D & 4 & 1.99 & Replication, recombination and repair \\
V & 5 & 2.49 & Cell cycle control, Cell division, chromosome partitioning \\
T & 9 & 4.48 & Defense mechanisms \\
N & 7 & 3.48 & Signal transduction mechanisms \\
C & 1 & 0.49 & Cell motility \\
G & 3 & 1.49 & Energy production and conversion \\
E & 14 & 6.97 & Carbohydrate transport and metabolism \\
F & 8 & 3.98 & Amino acid transport and metabolism \\
H & 4 & 1.99 & Nucleotide transport and metabolism \\
I & 6 & 2.99 & Coenzyme transport and metabolism \\
P & 1 & 0.49 & Lipid transport and metabolism \\
Q & 1 & 0.49 & Inorganic ion transport and metabolism \\
R & 1 & 0.49 & Secondary metabolites biosynthesis, transport and catabolism \\
S & 9 & 4.48 & General function prediction only \\
- & 3 & 1.49 & Function unknown \\
\hline & 116 & 57.71 & Not in CoGs \\
\hline
\end{tabular}


a

85 B. amyloliquefaciens subsp. plantarum UCMB5113(HG328254)

B. amyloliquefaciens subsp. amyloliquefaciens KHG19(CP007242)

68 B. amyloliquefaciens TF28

94 B. amyloliquefaciens subsp. plantarum TrigoCor1448 (CP007244)

83B. amyloliquefaciens G341 (CP011686)

B. amyloliquefaciens subsp. plantarum UCMB5033 (HG328253)

95. B. amyloliquefaciens subsp. plantarum $\mathrm{FZB} 42^{\mathrm{T}}(\mathrm{CP} 000560)$

B. amyloliquefaciens subsp. plantarum CC178 (CP006845)

87. B. amyloliquefaciens subsp. plantarum SQR9( CP006890)

98 B. amyloliquefaciens subsp. plantarum YAU B9601-Y2 (HE774679)

55 B. amyloliquefaciens subsp. plantarum NAU-B3(HG514500)

99 B. amyloliquefaciens subsp. plantarum CAU B946(HC617159)

98 B. amyloliquefaciens subsp. plantarum NJN-6( CP007165)

B. amyloliquefaciens LFB112 (CP006952)

B. amyloliquefaciens subsp. amyloliquefaciens $\mathrm{DSM}^{\mathrm{T}}(\mathrm{FN} 597644)$

97ЦB. amyloliquefaciens XH7(CP002927)

B. amyloliquefaciens LL3(CP002634)

B. amyloliquefaciens TA208 (CP002627)

100|B. amyloliquefaciens L-H15 (CP010556)

B. amyloliquefaciens L-S60 (CP011278)

100|B. amyloliquefaciens subsp. plantarum UCMB5036 (HF563562)

B. amyloliquefaciens $\mathrm{Y} 2(\mathrm{CP} 003332)$

B. amyloliquefaciens IT-45 (CP004065)

0.2

b

98 B. amyloliquefaciens subsp. amyloliquefaciens KHG19(CP007242)

98 B. amyloliquefaciens G341 (CP011686)

62 B. amyloliquefaciens subsp. plantarum UCMB5033 (HG328253)

39 B. amyloliquefaciens subsp. plantarum SQR9( CP006890)

39 B. amyloliquefaciens subsp. plantarum TrigoCor1448 (CP007244)

84 B. amyloliquefaciens subsp. plantarum $\mathrm{FZB} 42^{\mathrm{T}}$ ( $\left.\mathrm{CP} 000560\right)$

99 B. amyloliquefaciens subsp. plantarum CC178 (CP006845)

69 B. amyloliquefaciens subsp. plantarum UCMB5036 (HF563562)

87 B. amyloliquefaciens TF28

80 B. amyloliquefaciens subsp. plantarum NAU-B3(HG514500)

91 B. amyloliquefaciens Y2(CP003332)

99 B. amyloliquefaciens subsp. plantarum YAU B9601-Y2 (HE774679)

B. amyloliquefaciens subsp. plantarum NJN-6( CP007165)

99 B. amyloliquefaciens LFB112 (CP006952)

92 B. amyloliquefaciens subsp. plantarum CAU B946(HC617159)

84 B. amyloliquefaciens L-H15 (CP010556)

87 B. amyloliquefaciens L-S60 (CP011278)

B. amyloliquefaciens subsp. amyloliquefaciens $\mathrm{DSM} 7^{\mathrm{T}}$ (FN597644)

B. amyloliquefaciens LL3(CP002634)

93 B. amyloliquefaciens XH7(CP002927)

B. amyloliquefaciens TA208 (CP002627)

B. amyloliquefaciens IT-45 (CP004065)

0.1

Fig. 6 Phylogenetic trees based on $\operatorname{gr} A(\mathbf{a})$ and $r p o B(\mathbf{b})$. The GenBank accession numbers are shown in parentheses. Sequences were aligned using CLUSTALW, and phylogenetic inferences were constructed using the neighbor-joining method within the MEGA 5.10 software. Numbers at the nodes represent percentages of bootstrap values obtained by repeating the analysis 1000 times to generate a majority consensus tree. The scale bar indicates 0.2 (gyrA) and $0.1(r p o B)$ nucleotide change per nucleotide position, respectively 
(Additional file 3: Table S3, Additional file 4: Table S4, Additional file 5: Table S5, Additional file 6: Table S6). B. amyloliquefaciens TF28 possessed 3 CRISPR domains. The CRISPR length is $422 \mathrm{bp}$ with $81 \mathrm{bp}$ direct repeat (DR) sequences be separated by 5 spacers. No CRISPR associated gene was observed due to the incomplete genome sequence. B. amyloliquefaciens NAU-B3 had 1 CRISPR domains. The CRISPR length is 67 bp with $26 \mathrm{bp}$ DR sequences be separated by 1 spacer. B. amyloliquefaciens IT- 45 had 2 CRISPR domains. The CRISPR length is 129 bp with 37 bp DR sequences be separated by 1 spacer. The full-length sequence of protein-coding gene, DNA gyrase subunit A $(g y r A)$ and RNA polymerase subunit B $(r p o B)$ derived from 22 strains of $B$. amyloliquefaciens, were chosen to phylogenetic analysis. The neighbor-joining (NJ) phylogenetic tree revealed that strain TF28 with most of $B$. amyloliquefaciens subsp. plantarum clustered into the same group, which is distinct from the type strain B. amyloliquefaciens subsp. amyloliquefaciens DSM $7^{\mathrm{T}}$ (Fig. 6).

\section{Conclusions}

In this study, we characterized the genome of B. amyloliquefaciens TF28 isolated from soybean root. Strain TF28 was classified as B. amyloliquefaciens subsp. plantarum on comparative analysis of $16 \mathrm{~S}$ rRNA sequence, DNA gyrase subunit A (gyrA) and RNA polymerase subunit B $(r p o B)$ gene sequences. The genome of strain TF28 has the giant gene clusters that are linked with biocontrol, including non-ribosomal synthesis of the polyketides difficidin and bacillaene, the antifungal lipopetides surfactin, plipastatin, mycosubtilin, bacilysin and bacillibactin. Mycosubtilin and plipastatin synthesis gene clusters were only observed in strain TF28. Ubiquinone and other terpenoid-uquinoid synthesis, bacterial chemotaxis, biosynthsis of siderophore group nonribosomal peptides, antibiotic biosynthesis and noxious substance degradation pathways were found which reflected a high capacity of strain TF28 to promote plant growth, inhibit pathogens and support environment fitness. 201 specific genes are found in strain TF28 which provides information for further analysis of the strain function. The availability of the genome provides insights to better understand the biocontrol mechanisms and facilitate the utilization of the strain in the future.

\section{Additional files}

Additional file 1: Table S1. Strain ID Summary (DOCX 16 kb) Additional file 2: Table S2. Gene clusters of secondary metabolites synthesis in B.amyloliquefaciens TF28 and B.amyloliquefaciens subsp. plantarum FZB42 ${ }^{\top}$ (DOCX $13 \mathrm{~kb}$ )

Additional file 3: Table S3. General feature of genome from 22 strains of B. amyloliquefaciens (DOCX $17 \mathrm{~kb}$ )
Additional file 4: Table S4. GenBank Accession Summary (DOCX $15 \mathrm{~kb}$ )

Additional file 5: Table S5. Scientific Name Summary (DOCX $12 \mathrm{~kb}$ )

Additional file 6: Table S6. Reference Search Summary (DOCX 12 kb)

Additional file 7: Table S7. Annotation Summary (DOCX 16 kb)

\section{Acknowledgements}

We appreciate Lu Gao, Shenzhen Huada Gene Technology Co., Ltd. very much for her assistance in bioinformatics analysis. We are also grateful to Xiaoyong Wang, Institute of Microbiology, Heilongjiang Academy of Sciences, for her help in the paper writing

Funding

This work was supported by the grants from Heilongjiang Academy of Sciences for subject team planning (2014sw09) and subject special planning (YXK14ZSM15).

Authors' contributions

SZ prepared and wrote the manuscript, $J \mathrm{~L}$ and CS designed research, WJ characterized strain TF28, LQ performed bioinformatics analysis, XC and JH participated in the statistical analysis, $Y L$ and JC participated in the sequence alignment. All authors read and approved the final manuscript.

\section{Competing interests}

The authors declare that they have no competing interests.

\section{Author details}

${ }^{1}$ Institute of Microbiology, Heilongjiang Academy of Sciences, Harbin 150010, China. ${ }^{2}$ Institute of Advanced Technology, Heilongjiang Academy of Sciences, Harbin 150020, China. ${ }^{3}$ Heilongjiang Academy of Sciences, Harbin 150001, China.

Received: 15 January 2016 Accepted: 22 August 2016

Published online: 21 September 2016

\section{References}

1. Adnan N, Shahid M, Sarosh B, Johan M, Erik BR. Complete genome sequence of plant associated bacterium Bacillus amyloliquefaciens subsp. plantarum UCMB5033. Stand Genomic Sci. 2014;9:718-25. doi:10.4056/sigs.4758653.

2. Lefort F, Calmin G, Pelleteret P, Farinelli L, Osteras M, Crovadorea J. Whole-genome shotgun sequence of Bacillus amyloliquefaciens strain UASWS BA1, a bacterium antagonistic to plant pathogenic fungi. Genome Announcements. 2014:2:1-2. doi:10.1128/genomeA.00016-14.

3. Qin YX, Han YZ, Yu YQ, Shang QM, Zhang B, Li PL. Complete genome sequence of Bacillus amyloliquefaciens L-S60, a plant growth-promoting and antifungal bacterium. J Biotechnol. 2015;212:67-8. doi:10.1016/j.jbiotenc 2015.08.008.

4. Qin YX, Han YZ, Shang QM, Zhang B, Li PL. Complete genome sequence of Bacillus amyloliquefaciens L-H15, a plant growth promoting rhizobacteria isolated from cucumber seedling substrate. J Biotechnol. 2015;200:59-60. doi:10.1016/j.jbiotec.2015.02.020.

5. Borriss R, Chen XH, Ruecert C, Blom J, Becker A, Baumgarth B, et al. Relationship of Bacillus amyloliquefaciens clades associated with strains $D S M 7^{\top}$ and FZB42 ${ }^{\top}$ : a proposal for Bacillus amyloliquefaciens subsp. amyloliquefaciens subsp. nov. and Bacillus amyloliquefaciens subsp. plantarum subsp. nov. based on complete genome sequence comparisions. Int J Syst Evol Microbiol. 2011;61:1786-801. doi:10.1099/ijs.0.02267-0.

6. Zhang SM, Sha CQ, Wang YX, Li J, Zhao XY, Zhan XC. Isolation and characterization of antifungal endophytic bacteria from soybean. Microbiol Tong Bao China. 2008:35:1593-9.

7. Zhang SM, Wang YX, Meng LQ, Li J, Zhao XY, Cao X, et al. Isolation and characterization of antifungal lipopeptides produced by endophytic Bacillus amyloliquefaciens TF28. Afr J Microbiol Res. 2012;6:1747-55. doi:10.5897/ AJMR11.1025.

8. Kammara R, Praveen KS, Rajesh K, Kaneez FS. CTAB-mediated, single-step preparation of competent Escherichia coli, Bifidobacterium sp. and Kluyve romyces lactis cells. Meta Gene. 2014;2:807-18. doi:10.1016/j.mgene.2014. 10.002 . 
9. Delcher AL, Bratke KA, Powers EC, Salzberg SL. Identifying bacterial genes and endosymbiont DNA with Glimmer. Bioinformatics. 2007;23:673-9. doi:10.1093/bioinformatics/btm009.

10. Benson G. Tandem repeats finder: a program to analyze DNA sequences. Nucleic Acids Res. 1999;27:573-80.

11. Lagesen K, Hallin PF, Rødland E, Stærfeldt HH, Rognes T, Ussery DW. RNAmmer: consistent and rapid annotation of ribosomal RNA genes. Nucleic Acids Res. 2007;35:3100-8. doi:10.1093/nar/gkm160.

12. Lowe TM, Eddy SR. tRNAscan-SE: A Program for improved detection of transfer RNA genes in genomic sequence. Nucleic Acids Res. 1997;25:0955-64.

13. Gardner PP, Daub J, Tate JG, Nawrocki EP, Kolbe DL, Lindgreen S, et al. Rfam: updates to the RNA families database. Nucleic Acids Res. 2009;37:D136-40. doi:10.1093/nar/gkn766.

14. Zhou Y, Liang YJ, Lynch K, Dennis JJ, Wishart DS. PHAST: A fast phage search tool. Nucleic Acids Res. 2011;39:W347-52. doi:10.1093/nar/gkr485.

15. Grissa I, Vergnaud G, Pourcel C. CRISPRFinder: a web tool to identify clustered regularly interspaced short palindromic repeats. Nucleic Acids Res. 2007;3:W52-7. doi:10.1093/nar/gkm360.

16. Bard J, Winter R. Gene Ontology: tool for the unification of biology. Nat Genet. 2000;25:25-9. doi:10.1038/75556.

17. Kanehisa M, Goto S, Hattori M, Aoki-Kinoshita KF, Itoh M, Kawashima S, et al. From genomics to chemical genomics: new developments in KEGG. Nucleic Acids Res. 2006;34:D354-7. doi:10.1093/nar/gkj102.

18. Tatusov RL, Fedorova ND, Jackson JD, Jacobs AR, Kiryutin B, Koonin EV, et al. The COG database: an updated version includes eukaryotes. BMC Bioinformatics. 2003;4:41. doi:10.1186/1471-2105-4-41.

19. Magrane M. UniProt C. UniProt Knowledgebase: a hub of integrated protein data. Database (Oxford). 2011: bar009. doi:10.1093/database/bar009

20. Bateman A, Coin L, Durbin R, Finn RD, Hollich V, Griffiths-Jones S, et al. The Pfam protein families database. Nucleic Acids Res. 2004;32:D138-41. doi:10.1093/nar/gkh121.

21. Kurtz S, Phillippy AL, Delcher AL, Smoot M, Shumway M, Antonescu C, et al. Versatile and open software for comparing large genomes. Genome Biol. 2004;5(2):R12. doi:10.1186/gb-2004-5-2-r12.

22. Qin J, Li R, Rase J, Arumugam M, Burgdorf KS, Manichanh C, et al. A human gut microbial gene catalogue established by metagenomic sequencing. Nature. 2010;464:59-65. doi:10.1038/nature08821.

23. Chen XH, Koumoutsi A, Scholz R, Eisenreich A, Schneider K, Heinemeyer I, et al. Comparative analysis of the complete genome sequence of the plant growth-promoting bacterium Bacillus amyloliquefaciens FZB42. Nat Biotechnol. 2007;25:1007-14. doi:10.1038/nbt1325.

24. Hossain MJ, Ran C, Liu K, Ryu CM, Rasmussen-lvy CR, Williams MA, et al. Deciphering the conserved genetic loci implicated in plant disease control through comparative genomics of Bacillus amyloliquefaciens subsp. plantarum. Front Plant Sci. 2015;6:631. http://www.frontiersin.org. Accessed 17 Aug 2015.

25. Niazi A, Manzoor S, Asari S, Bejai S, Meijer J, Bongcam-Rudloff E. Genome Analysis of Bacillus amyloliquefaciens Subsp. plantarum UCMB5113: A rhizobacterium that improves plant growth and stress management. PLoS One. 2014;9:1-14. doi:10.1371/journal.pone.0104651.

26. Field D, Garrity G, Gray T, Morrison N, Selengut J, Sterk P, et al. The minimum information about a genome sequence (MIGS) specification. Nature Biotechnol. 2008:26:541-7. doi:10.1038/nbt1360.

27. Woese CR, Kandler O, Wheelis ML. Towards a natural system of organisms: proposal for the domains Archaea, Bacteria, and Eucarya. Proc Natl Acad Sci U S A. 1990;87:4576-9. doi:10.1073/pnas.87.12.4576.

28. Gibbons NE, Murray RGE. Proposals concerning the higher taxa of bacteria. Int J Syst Bacteriol. 1978;28:1-6. doi:10.1099/00207713-28-1-1.

29. Skerman VBD, McGowan V, Sneath PHA. Approved lists of bacterial names. Int J Syst Bacteriol. 1980:30:225-420. doi:10.1099/00207713030-1-225.

30. Murray RGE. The higher taxa, or, a place for everything...? In: Crieg NR, Holt JG, editors. Bergey's manual of systematic bacteriology, vol. 1. 1st ed. Baltimore: The williams and wilkins co.; 1984. p. 31-4.

31. Euzéby J. List of new names and new combinations previously effectively, but not validly, published. List no. 132. Int J Syst Evol Microbiol. 2010;60:469-72 doi:10.1099/ijs.0.022855-0.

32. Ludwig W, Schleifer KH, Whitman WB. Class I. Bacilli class nov. In: De Vos P, Garrity G, Jones D, Krieg NR, Ludwig W, Rainey FA, Schleifer KH, Whitman WB, editors. Bergey's manual of systematic bacteriology, vol. 3. 2nd ed. New York: Springer; 2009. p. 19-20.
33. Prévot AR, Hauderoy P, Ehringer G, Guillot G, Magrou J, Prevot AR, et al. editors. Dictionnaire des Bactéries Pathogènes. 2nd ed. Paris: Masson et Cie; 1953. p. 1-692

34. Fischer A. Untersuchungen über Bakterien. Jahrbücher für Wissens chaftliche Botanik. 1895;27:1-163.

35. Cohn F. Untersuchungen über Bakterien. Beitr Biol Pflanz. 1872;1:127-224.

36. Priest FG, Goodfellow M, Shute LA, Berkeley RCW. Bacillus amyloliquefaciens sp. nov., nom. rev. Int J Syst Bacteriol. 1987;37:69-71.

37. Wang LT, Lee FL, Tai CJ, Kuo HP. Bacillus velezensis is a later heterotypic synonym of Bacillus amyloliquefaciens. Int J Syst Evol Microbiol. 2008;58:671-5. doi:10.1099/ijs.0.65191-0.

38. Dunlap CA, Kim SJ, Kwon SW, Rooney AP. Phylogenomic analysis shows that Bacillus amyloliquefaciens subsp. plantarum is a later heterotypic synonym of Bacillus methylotrophicus. Int J Syst Evol Microbiol. 2015;65:2104-9. doi:10.1099/ijs.0.000226.

39. Ashburner M, Ball CA, Blake JA, Botstein D, Butler H, Cherry JM, et al. Gene Ontology: tool for the unification of biology. Nat Genet. 2000;25:25-9. doi:10.1038/75556.

\section{Submit your next manuscript to BioMed Central and we will help you at every step:}

- We accept pre-submission inquiries

- Our selector tool helps you to find the most relevant journal

- We provide round the clock customer support

- Convenient online submission

- Thorough peer review

- Inclusion in PubMed and all major indexing services

- Maximum visibility for your research

Submit your manuscript at www.biomedcentral.com/submit
) Biomed Central 